\title{
Coffee consumption and risk of colorectal cancer in a population based prospective cohort of Swedish women
}

P Terry, L Bergkvist, L Holmberg, A Wolk

\begin{abstract}
Background-The presumed protective effect of coffee consumption on colorectal cancer, which is supported by case control studies, has not been confirmed in prospective cohort studies. Cohort studies are few in number however and often suffer from a small number of cases, limited attention to confounding variables, and a low percentage of heavy coffee drinkers. Methods-We examined data from a large population based cohort of Swedish women who were free from cancer at the start of follow up, with a wide range of coffee consumption, information on many potentially confounding variables, and a larger number of cases than any previous cohort study of coffee consumption and colorectal cancer.
\end{abstract}

Results-During an average of 9.6 years of follow up of 61463 women aged 40-74 years, we observed 460 incident cases of colorectal cancer (291 with colon cancer, 159 with rectal cancer, 10 with cancer at both sites). We found no association between coffee consumption and colorectal cancer risk. The risk ratio for drinking four or more cups per day compared with none was $1.04(95 \%$ confidence interval $0.63-1.69 ; \mathrm{p}$ for trend 0.84 ). The findings were similar for cancers of the distal and proximal colon and rectum.

Conclusions-The recently published affirmative conclusions regarding the protective effect of coffee consumption may be premature. For patients seeking advice about coffee consumption, the evidence suggests that moderate or even high consumption will probably not influence the risk of colorectal cancer.

(Gut 2001;49:87-90)

Department of Surgery and Centre for Clinical Research, Uppsala University, Västerås, Sweden

L Bergkvist

Regional Oncologic Centre, Uppsala, Sweden

L Holmberg

Correspondence to: P Terry, Department of Medical Epidemiology, Karolinska Institute, Box 281, 17177 Stockholm, Sweden

paul.terry@mep.ki.se

Accepted for publication 17 January 2001 Evidence from the majority of studies of the relation between coffee consumption and the risk of colorectal cancer has led some investigators to conclude that the data convincingly support a protective effect. ${ }^{1}$ As coffee is a common exposure, and colorectal cancer is among the most common diagnosed cancers in developed countries, ${ }^{2}$ this issue is of public health importance. In a recent meta-analysis, ${ }^{3}$ case control studies generally showed consistent inverse associations while cohort studies did not support a protective effect. Cohort studies that address this association are few however and often suffer from a small number of cases and limited attention to potentially confounding variables. ${ }^{3}$ Moreover, pooling of results by geographic region showed stronger inverse associations among Northern European studies compared with studies conducted in Southern Europe and in the USA. ${ }^{3}$ This geographic difference is consistent with the higher range of coffee consumption in Scandinavia ${ }^{4}$ and suggests the possibility that the range of exposure to coffee may be too narrow in some of the cohort studies to detect a true protective effect.

We examined data from a large population based prospective cohort of women in Sweden with a wide range of coffee consumption, information on many potentially confounding variables, and a larger number of cases than any previous study of coffee consumption and colorectal cancer.

\section{Methods}

THE SWEDISH MAMMOGRAPHY SCREENING COHORT

From 1987 to 1990 , a population based mammography screening programme was introduced in two counties in central Sweden. In Västmanland county all women born between 1917 and 1948 received a mailed invitation to be screened by mammography between March 1987 and March 1989 ( $n=41$ 786) together with a six page questionnaire; 31735 women $(76 \%)$ returned completed questionnaires. In Uppsala county all women born between 1914 and 1948 were invited to the screening and received the same questionnaire between January 1988 and December $1990(\mathrm{n}=48$ 517); 34916 women $(72 \%)$ returned completed questionnaires. Hence questionnaires completed before the mammography were obtained from $66651(73.8 \%)$ women in the source population. These questionnaires included items on age, weight $(\mathrm{kg})$, height $(\mathrm{cm})$, education, and diet.

For the present analyses, we excluded

women who were outside the age range 40-74 years $(n=165)$, those with missing $(n=707)$ or incorrect ID numbers $(n=415)$, as well as those with no date on the questionnaire $(n=608)$, a missing date as a result of moving out of the study area $(n=79)$, or no date of death $(n=16)$. After further exclusion of 793 women with extreme energy intake estimates (below or above mean \pm 3 SD for $\log _{\mathrm{e}}$ transformed calories), the cohort was restricted to 63868 women. By linkage to the Swedish Cancer Registry, we identified and excluded all women 
with a previous cancer diagnosis other than non-melanoma skin cancer $(n=2405)$. Thus the study cohort comprised 61463 women at the start of follow up.

DIETARY ASSESSMENT

Participants were asked how often over the past six months, on average, they had consumed coffee and 66 other commonly eaten foods. For coffee consumption, eight predefined frequency categories were used including: "never/ seldom," " $1-3$ cups per month," "one cup per week," "2-3 cups per week," " $4-6$ cups per week," "one cup per day," "2-3 cups per day," and "four or more cups per day." For nutrient calculations, age specific portion sizes were used $(40-52,53-65,66-74$ years) based on mean values from 5922 days of weighed food records among 213 women randomly selected from the study population. Intake of nutrients was computed by multiplying the frequency of consumption of each unit of food by the nutrient content of the specified portions, as per the Swedish National Food Administration data. ${ }^{5}$ The validity of dietary assessment based on self reported food frequencies was evaluated in a subsample of 129 women from the cohort. During four, seven day periods, 3-4 months apart, each participant weighed and recorded all foods consumed. The validity estimate for coffee consumption was measured as a Pearson correlation coefficient between the food questionnaire and food records $(r=0.54)$.

IDENTIFICATION OF COLON AND RECTUM CANCER CASES AND FOLLOW UP OF THE COHORT

Cases of invasive colon and rectum cancers were identified through matching of the cohort with the computerised regional cancer registers of colon and rectal cancer diagnoses in the two counties to 31 December 1998. Quality control of the regional cancer registers was performed by a search for cases through the departments of pathology in the region and a study of all reported individual medical records during the follow up period of 1987-1994. The completeness of follow up for colorectal cancers was documented to be $98 \%$, as was the completeness of the National Cancer Registry as a whole. ${ }^{6}$ Colon cancers were defined as those occurring above the peritoneal delineation of the abdominal cavity and rectal cancers were those occurring below this delineation. Proximal colon cancers were defined as those occurring from the caecum to the splenic flexure $(n=118)$, and distal colon cancers were defined as those occurring from the descending colon to the sigmoid colon $(n=101) ; 72$ were of unspecified location. Date of death in the cohort was ascertained through the Swedish Death Register and date of moving away from the study area was obtained by matching the cohort with the computerised and continuously updated Swedish Population Register. The study was approved by the ethics committee at Uppsala University Hospital and by the Karolinska Institute's regional ethics committee.

\section{STATISTICAL ANALYSIS}

Cox proportional hazards models were used to estimate hazard rate ratios with $95 \%$ confidence intervals (CI) relating different explanatory variables to the occurrence of invasive colorectal cancer. Follow up was censored at date of death, date of migration out of the study area, or at the end of the follow up period (31 December 1998). Multivariate models included age in five year age groups, body mass index (quartiles), education level (less than high school, high school, and university), quartiles of intakes of energy, total fat, dietary fibre, calcium, vitamin C, folic acid, vitamin D, red meat, and alcohol. As a basis for the trend tests, scores were constructed from the median values of the categorised variables.

\section{Results}

During an average of 9.6 years of follow up of our cohort of 61463 women (588 270 person years) we observed 460 incident cases of colorectal cancer (291 colon, 159 rectal, 10 with both colon and rectal cancer). Mean age at diagnosis of colon and rectal cancer was 67 and 68 years, respectively. Slightly more than $18 \%$ of our population consumed four or more cups of coffee per day, and approximately $78 \%$ consumed two or more cups per day (table 1). Coffee consumption was associated with higher intakes of calories, red meat, calcium, and alcohol, and with lower intakes of vitamin $\mathrm{C}$ and folic acid. Median age of subjects in the highest coffee consumption category was slightly lower than the median age of those

Table 1 Baseline characteristics of the study cohort

\begin{tabular}{|c|c|c|c|c|}
\hline \multirow[b]{2}{*}{ Characteristic ${ }^{\star}$} & \multicolumn{4}{|c|}{ Coffee consumption } \\
\hline & $\begin{array}{l}<1 \text { cup/day } \\
(n=5537)\end{array}$ & $\begin{array}{l}1 \text { cup/day } \\
(n=8278)\end{array}$ & $\begin{array}{l}2-3 \text { cups/day } \\
(n=36576)\end{array}$ & $\begin{array}{l}4+\text { cups/day } \\
(n=11\end{array}$ \\
\hline Person years & 52340 & 78545 & 351223 & 106161 \\
\hline Median age at baseline (y) & 53 & 57 & 53 & 48 \\
\hline Median body mass index $\left(\mathrm{kg} / \mathrm{m}^{2}\right)$ & 24.1 & 24.4 & 24.2 & 24.0 \\
\hline \multicolumn{5}{|l|}{ Dietary intake (median) } \\
\hline Energy (kcal/day) & 1216 & 1227 & 1310 & 1374 \\
\hline Total fat (g/day) & 44.4 & 44.2 & 45.1 & 46.4 \\
\hline Dietary fibre (g/day) & 17.2 & 17.3 & 16.9 & 16.1 \\
\hline Calcium (mg/day) & 725 & 732 & 738 & 749 \\
\hline Vitamin C (mg/day) & 63.4 & 65.8 & 61.3 & 56.3 \\
\hline Folic acid ( $\mu \mathrm{g} /$ day) & 196 & 194 & 187 & 179 \\
\hline Vitamin $\mathrm{D}(\mu \mathrm{g} /$ day $)$ & 3.2 & 3.3 & 3.4 & 3.4 \\
\hline Red meat (servings/week) & 4.1 & 4.1 & 4.6 & 5.1 \\
\hline Alcohol (g/day) & 1.5 & 1.8 & 2.2 & 2.6 \\
\hline
\end{tabular}

*Values presented are age standardised. 
Table 2 Relation between coffee consumption and colorectal cancer ${ }^{\star}$

\begin{tabular}{llllll}
\hline & $\begin{array}{l}\text { Colorectal } n=460 \\
(R R(95 \% C I))\end{array}$ & $\begin{array}{l}\text { Colon } n=291 * * \\
(R R(95 \% C I))\end{array}$ & $\begin{array}{l}\text { Proximal colon } n=118 \\
(\text { RR }(95 \% C I))\end{array}$ & $\begin{array}{l}\text { Distal colon } n=101 \\
(\text { RR }(95 \% \text { CI }))\end{array}$ & $\begin{array}{l}\text { Rectal } n=159 \\
(R R(95 \% C I))\end{array}$ \\
\hline$<1$ cup/day & 1.0 (reference) & 1.0 (reference) & 1.0 (reference) & 1.0 (reference) & $1.0($ reference) \\
1 cup/day & $0.96(0.66-1.40)$ & $0.97(0.61-1.54)$ & $0.89(0.45-1.79)$ & $0.84(0.39-1.81)$ & $0.88(0.45-1.73)$ \\
$2-3$ cups/day & $0.93(0.67-1.29)$ & $0.89(0.59-1.33)$ & $0.80(0.44-1.46)$ & $0.78(0.41-1.50)$ & $1.01(0.57-1.78)$ \\
$4+$ cups/day & $1.04(0.70-1.54)$ & $1.06(0.65-1.72)$ & $1.17(0.56-2.43)$ & $0.94(0.43-2.08)$ & $1.06(0.54-2.10)$ \\
p for trend & 0.95 & 0.97 & 0.94 & 0.80 & 0.69 \\
\hline
\end{tabular}

* Rate ratios (RR) with $95 \%$ confidence interval (CI).

Multivariate models included age in five year age groups, body mass index (quartiles), education level (less than high school, high school, and university), quartiles of intakes of energy and red meat, and quartiles of energy adjusted total fat, dietary fibre, calcium, vitamin $\mathrm{C}$, folic acid, vitamin $\mathrm{D}$, and alcohol.

$\star \star 72$ cases of colon cancer were of unspecified location.

consuming less. There was no appreciable difference in median fat intake or body mass index among coffee consumption groups.

Coffee consumption was not associated with a lowered risk of colorectal cancer in age adjusted or multivariate adjusted models (table 2 ). Eight original coffee consumption groups were collapsed into four due to the small number of cases in some exposure categories. In the collapsed data, the age adjusted rate ratio for the highest exposure (4+ cups/day) compared with the lowest ( $<1$ cup/day) was 1.09 (95\% CI $0.74-1.61 ; \mathrm{p}$ for trend 0.73 ) for colorectal cancer and the multivariate adjusted rate ratio was 1.04 (95\% CI $0.70-1.54$; p for trend 0.95$)$. Using the original eight categories, the age adjusted rate ratio for the highest exposure $(4+$ cups/day) compared with the lowest (never or seldom) was 1.11 (95\% CI 0.68 $1.81 ; \mathrm{p}$ for trend 0.97 ), and the multivariate adjusted rate ratio was 1.04 (95\% CI $0.63-$ 1.69; $\mathrm{p}$ for trend 0.84 ). Similarly, we found no association for colon cancer (total, proximal, or distal) or rectal cancer in the original or collapsed data (multivariate adjusted data shown). Examined over strata of age (in five year age groups), body mass index (quartiles), and energy adjusted intakes of alcohol, fat, fibre, folic acid, vitamin $\mathrm{C}$, and vitamin $\mathrm{D}$ (quartiles), no differences in the association between coffee consumption and colorectal cancer were observed (not shown).

In additional analyses, the multivariate adjusted rate ratio for the highest quartile of total caffeine intake compared with the lowest was 1.10 (95\% CI 0.80-1.51; p for trend 0.91). Tea consumption showed a non-significant positive association with colorectal cancer risk but exposure to tea was relatively low compared with coffee. To eliminate the possibility that changes in coffee consumption due to preclinical undiagnosed colorectal cancers could influence our results, we performed an analysis after excluding cases that occurred within the first three years of follow up. This exclusion did not alter our results.

\section{Discussion}

In our population based prospective cohort, with a wide range of coffee consumption and the largest number of cases studied to date, we found no association between coffee consumption and risk of total colorectal cancer, proximal or distal colon cancer, or rectal cancer. Regular consumers of four or more cups of coffee per day showed no decreased risk of colorectal cancer compared with those who never or rarely drank coffee. Statistical adjustment for potentially confounding variables did not alter this finding. In subgroup analyses, we found no evidence that the association with coffee consumption varied over strata of age, body mass index, or various dietary factors.

In a recent meta-analysis, ${ }^{3}$ the calculated relative risk for "high" versus "low" coffee consumption in five cohort studies with number of cases ranging from 126 to 269 was 0.97 (95\% CI 0.73-1.29; p for trend 0.83). Conversely, the calculated relative risk for 12 case control studies, including a Swedish study, ${ }^{7}$ was 0.72 (95\% CI 0.61-0.84; $\mathrm{p}$ for trend <0.001). The calculated relative risk for 17 studies of both cohort and case control design was 0.76 (95\% CI $0.66-0.89$; $\mathrm{p}$ for trend $<0.001)$, reflecting the inverse associations of the more numerous case control studies. The reasons for the differing results by study design are unknown but may be related to changes in coffee consumption among cases due to the adverse effects on digestion..$^{8-10}$ In addition to our study, the results of another prospective cohort study, with 185 colorectal cancer cases, reported after the meta-analysis showed no significant association between coffee consumption and colon or rectal cancer. ${ }^{11}$

It seems unlikely that differences in results by study design can be explained by differences in coffee brewing method. During the time period in which percolation or boiling have ceased to be popular methods for brewing coffee, results from case control studies show no clear trend in association between coffee consumption and colorectal cancer risk. ${ }^{3}$ Moreover, assessment of coffee consumption over the same time period that our data were collected showed an inverse association in a previous case control study from the same region in Sweden, ${ }^{7}$ where we would suspect brewing methods are comparable. Boiling coffee, as opposed to use of a coffee filter, was prevalent 10 years ago in Sweden only among inhabitants of the most northern areas. ${ }^{12}$ Significant inverse associations have also been seen in case control studies from the USA ${ }^{13}$ and Italy ${ }^{14}$ during periods where non-filtered coffee consumption was presumed to be rare..$^{1516}$ However, brewing method in relation to colorectal cancer has not been formally studied and further examination of this issue might be informative.

The strengths of our study include the large sample size of our cohort and its population 
based design, specific subsite diagnoses for the majority of colorectal cancer cases, and completeness of follow up in the Swedish cancer register system. It is unlikely that undiagnosed early stages of colon or rectum cancer altered coffee consumption as we also observed no association after excluding cancer cases that occurred during the first three years of follow up. Moreover, the large number of cases allowed us to examine associations among subgroups of our population and subsites of colorectal cancer with reasonable statistical power. The prospective assessment of exposure in our study eliminates information bias from selective recall, a potential threat to the validity of case control studies. Our data were limited however by the likelihood of some degree of measurement error of exposure. Such nondifferential misclassification would tend to attenuate relative risk estimates ${ }^{17}$ but does not easily explain the discrepancy between cohort studies and case control studies. We cannot rule out a weak effect of coffee consumption, although the consistency of our findings with those of other cohort studies suggests that a strong or even moderate protective effect is unlikely.

We could not adjust our relative risk estimates for exercise as this information was not collected at baseline. Energy intake, an approximate indicator of physical activity ${ }^{18}$ was not associated with colorectal cancer in our data and our results were not altered by adjustment for the effects of energy intake or body mass index. Moreover, the similar median value for body mass index and higher median energy intake in the highest coffee consumption category compared with the lowest indicates that physical activity was, if anything, higher among subjects drinking the most coffee.

On the other hand, our 67 item food frequency questionnaire may not allow estimation of energy intake with great precision, and thus adjustment for total caloric intake may not fully account for between person variation due to energy intake or physical activity. Therefore, some residual confounding by these factors may exist. Our data were also limited by lack of information on smoking. Smoking has not previously been associated with colorectal cancer risk in Swedish women, perhaps due to their low rate of long term smoking. In a population based assessment of smoking that covered the period $1967-1971,{ }^{19}$ only 23 of over 10000 women smoked more than 20 cigarettes per day, and no association was observed with colorectal cancer in this group. Furthermore, statistical control for smoking did not alter the negative results for coffee in a prospective study from Norway, ${ }^{20}$ nor was a statistically significant association observed for coffee consumption in another prospective cohort study that controlled for smoking. ${ }^{21}$ However, we cannot rule out the possibility of some confounding due to smoking in our data.

In conclusion, our findings, based on a large population based cohort study with a wide range of coffee consumption and the largest number of cases examined to date, do not support an inverse association with colorectal cancer. Thus the recently published affirmative conclusions regarding the protective effect of coffee consumption ${ }^{1}$ may be premature. Increasingly, the public is aware of the potential role of dietary factors, such as coffee, in disease aetiology. For patients seeking advice about coffee consumption, the evidence suggests that moderate or even high consumption will not likely influence the risk of colorectal cancer.

This study was supported by grants from the Swedish Council for Planning and Coordination of Research and from the Swedish Cancer Society.

1 Ekbom A. Substantial coffee consumption was associated with a lower risk of colorectal cancer in the general population. Gut 1999; 44:597.

2 Parkin D. Estimates of the worldwide incidence of 25 major cancers in 1990. Int $\mathcal{f}$ Cancer 1999;80:827-41.

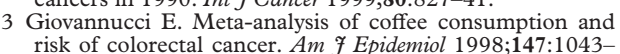
52.

4 Fredholm B, Bättig K, Holmén J, et al. Actions of caffeine in the brain with special reference to factors that contribute to its widespread use. Pharmacol Rev 1999;51:83-133.

5 Bergström L, Kylberg E, Hagman U, et al. The food composition database KOST: the National Food Administration's information system for nutritive values of food. Vår Föda 1991;43:439-47.

6 Mattsson B, Wallgren A. Completeness of the Swedish Cancer Registry: non-notified cancer cases recorded on death certificates in 1978. Acta Radiol Oncol 1984;23:305-13.

7 Baron J, Gerhardsson de Vergier M, Ekbom A. Coffee, tea, tobacco, and cancer of the large bowel. Cancer Epidemiol Biomarkers Prev 1994;3:565-70.

8 Babb R. Coffee, sugars, and chronic diarrhea: why a diet history is important. Postgrad Med 1984;75:82, 86-7.

9 Sander R, Jordan M, Shelton B. Demographic and dietary determinants of constipation in the US population. $A m \mathcal{F}$ Public Health 1990;80:185-9.

10 Hill P. It is not what you eat, but how you eat it: digestion, lifestyle, nutrition. Nutrition 1991;7:385-95.

11 Hartman T, Tangrea J, Pietinin P, et al. Tea and coffee consumption and risk of colon and rectal cancer in middle-aged Finnish men. Nutr Cancer 1998;31:41-8

2 Lindahl B, Johansson I, Huhtasaari F, et al. Coffee drinking and blood cholesterol - effects of brewing methods, food intake and life style. F Intern Med 1991;230:299-305.

13 Rosenburg L, Werler MM, Palmer JR, et al. The risks of cancers of the colon and rectum in relation to coffee consumption. Am f Epidemiol 1989;130:895-903.

14 Centonze S, Boeing H, Leoci C, et al. Dietary habits and colorectal cancer in a low-risk area: results from a population-based case-control study in southern Italy. Nutr Cancer 1994:21:23.46.

15 Greenland S. A meta-analysis of coffee, myocardial infarction, and coronary death. Epidemiology 1993;4:366-

16 Salvaggio A, Periti M, Miano L, et al. Coffee and cholesterol, an Italian study. Am f Epidemiol 1991;134:14956.

17 Rothman KJ, Greenland S. Precision and validity in epidemiologic studies. In: Modern epidemiology, 2nd ed. Philadelphia: Lippincott, 1998:127-132, 53-6.

18 Willett W, Howe G, Kushi L. Adjustment for total energy intake in epidemiologic studies. Am f Clin Nutr 1997; 65(suppl 4):1220-8S.

19 Terry P, Ekbom A, Lichtenstein P, et al. Long-term tobacco smoking and colorectal cancer in a prospective cohort study. Int $\mathcal{F}$ Cancer 2001;91:585-7.

20 Stensvold I, Jacobsen BK. Coffee and cancer: a prospective study of 43,000 Norwegian men and women. Cancer Causes Control 1994;5:401-8.

21 Klatsky AL, Armstrong MA, Friedman GD, et al. The relations of alcoholic beverage use to colon and rectal cancer. Am F Epidemiol 1988;128:1007-15. 